SCIENTIFIC REPORT

\title{
Ophthalmodynamometric determination of the central retinal vessel collapse pressure correlated with systemic blood pressure
}

\section{J B Jonas}

Aims: To evaluate whether determination of the central retinal artery and vein collapse pressure correlate with systemic blood pressure measurements, using a new Goldmann contact lens associated ophthalmodynamometric device

Methods: The prospective clinical study included 92 eyes of 92 patients presenting with cataract or refractive problems ( $n=40$; control study group) or with retinal and orbital pathologies $(n=52)$. With topical anaesthesia, a Goldmann contact lens fitted with a pressure sensor in its holding ring was placed onto the cornea. Pressure was asserted onto the globe by pressing the contact lens, and the pressure value at the time when the central retinal artery and vein started pulsating were noted as central retinal artery and vein collapse pressure. Additionally, the brachial arterial blood pressure was measured.

Results: In the control study group, central retinal artery collapse pressure was highly significantly correlated with diastolic blood pressure (correlation coefficient $r=0.77$; $p<0.001)$ and systolic blood pressure $(r=0.35 ; p=0.03)$. Central retinal vein collapse pressure was statistically independent of diastolic blood pressure $(p=0.11)$. In eyes with retinal or orbital diseases, the correlation coefficients were lower than in the control study group. In eyes with retinal arterial occlusions, central retinal vessel collapse pressure measurements were not correlated with arterial blood pressure measurements.

Conclusions: Depending on coexisting retinal or orbital diseases, ophthalmodynamometric estimation of the central retinal artery collapse pressure, performed during a routine Goldmann contact lens ophthalmoscopy, correlates with systemic blood pressure measurements.

E xamination of the central retinal artery and vein can have importance for the diagnosis of ocular, orbital, and neurological diseases because of their intimate anatomical relationship with the cerebrovascular system, the orbit, and the cerebrospinal fluid system. ${ }^{1-25}$ Previous studies have suggested that the collapse pressure of the central retinal artery, as measured by a new ophthalmodynamometric device, is abnormally low in patients suffering from ischaemic ophthalmopathy. ${ }^{17}$ The collapse pressure of the central retinal vein is increased in patients with increased cerebrospinal fluid pressure, ${ }^{711} 141518$ in patients with chronic open angle glaucoma, ${ }^{23}$ in patients with retinal venous stasis or retinal vein occlusions, ${ }^{19}$ in patients with spontaneous carotid cavernous sinus fistula, ${ }^{21}$ as well as in patients with thyroid associated orbitopathy. ${ }^{24}$ Based on these previous studies, the purpose of the present investigation is to evaluate whether the systemic blood pressure influences the collapse pressure of the central retinal vessels. The results may be important for the discussion and interpretation of ophthalmodynamometric examinations, by addressing the question of whether systemic blood pressure should generally be taken into account if ophthalmodynamometric measurements are taken; the results may show whether the systemic blood pressure can be estimated during a routine ophthalmoscopic examination using a new ophthalmodynamometric device.

\section{PATIENTS AND METHODS}

The total population of the prospective clinical study included 92 eyes of 92 patients (51 women) who were divided into three study groups.

The control study group consisted of 40 eyes of 40 subjects (27 women) with a mean age of 68.7 (SD 13.3) years (range 37.0-95.1 years) who attended the hospital because of refractive problems or cataract.

The second study group included 41 eyes of 41 patients (21 women) with a mean age of 67.2 (SD 12.9) years (range 23.7-84.0 years). The study group was divided into two subgroups. The glaucoma subgroup consisted of 21 eyes of 21 patients with primary or secondary open angle glaucoma (age 68.0 (SD 11.9) years). The non-glaucomatous subgroup included 20 patients (age 64.8 (SD 14.5) years) with retinal or orbital diseases in which the retinal arterial perfusion was not completely reduced, such as proliferative diabetic retinopathy, retinal venous stasis syndrome, and endocrine orbitopathy.

The third study group consisted of 11 eyes of 11 patients ( 3 women; mean age 72.5 (SD 12.6) years; range 60.8-99.8 years) with retinal arterial occlusive diseases such as central retinal artery occlusion, ischaemic central retinal vein occlusion, branch retinal artery occlusion, and ischaemic ophthalmopathy.

In the whole study population, refractive error ranged between -7 diopters and +5 diopters. Intraocular pressure (IOP) ranged between $12 \mathrm{~mm} \mathrm{Hg}$ and $21 \mathrm{~mm} \mathrm{Hg}$. The university's ethics committee had approved that the methods applied in the study adhered to the tenets of the Declaration of Helsinki for the use of human subjects in biomedical research. Informed consent was obtained from each patient before enrolment into the study. The three study groups did not vary significantly in mean diastolic blood pressure (78.3 (SD 12.9) mm Hg $v 81.2$ (11.8) mm Hg $v 79.1$ (17.0) mm Hg $(\mathrm{p}>0.60)$ ) or systolic blood pressure (145.0 (15.9) mm Hg $v$ 147.0 (20.6) mm Hg $v 153.6$ (23.4) mm Hg; p>0.20)).

For all patients included in the study, a ophthalmodynamometry was performed. The ophthalmodynamometer

Abbreviations: IOP, intraocular pressure 
(Meditron GmbH, Völklingen, Germany) consisted of a conventional Goldmann contact lens fitted with a pressure sensor ring at its outer margin where the Goldmann contact lens is usually held during an ophthalmoscopic examination. The ophthalmodynamometric measurements are given in relative units. After medical mydriasis, the Goldmann contact lens of the ophthalmodynamometer was placed onto the corneal surface with topical anaesthesia. Slightly applying increasing pressure onto the contact lens, the optic nerve head was continuously observed biomicroscopically. When the central retinal artery or its branches on the surface of the optic nerve head showed early pulsations, the pressure measured by the sensor ring in the Goldmann contact lens was noted. When, in an independent and second step of the examination, the central retinal vein or one of its branches on the optic disc surface started to show pulsations, the value given by the pressure sensor was noted as the central retinal vein collapse pressure. All measurements were repeated nine times. The mean of the ten measurements was taken for further statistical analysis. The right eye was always measured before the left eye. The method has already been described in detail. ${ }^{17-25}$ As the pressure exerted onto the central retinal vessels to make them collapse is the sum of IOP plus the pressure by the ophthalmodynamometric contact lens on the cornea, we calculated an additional parameter as the artificial sum of intraocular pressure (mm $\mathrm{Hg})$ plus the ophthalmodynamometric measurement (relative units).

Blood pressure was measured after the examination. Systolic blood pressure and diastolic blood pressure were determined using a mercury sphygmomanometer. Mean arterial blood pressure was defined as diastolic blood pressure plus one third of the difference of systolic blood pressure minus diastolic blood pressure.

In the statistical analysis, only one randomly selected eye per subject and patient was taken for statistical analysis. For intraindividual bilateral comparisons, only subjects and patients with bilateral examinations were considered.

\section{RESULTS}

In the eyes of the control study group, the diastolic collapse pressure of the central retinal artery was highly significantly

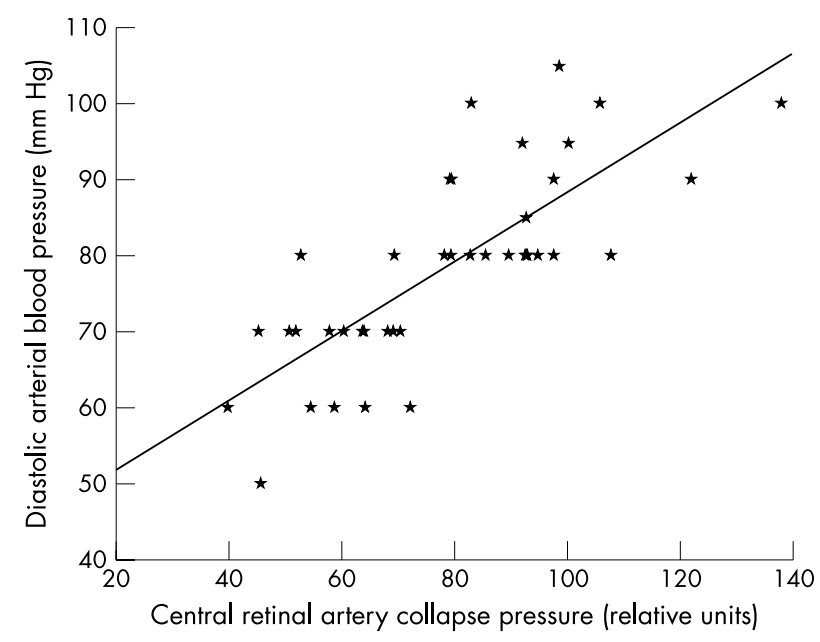

Figure 1 Scattergram showing the correlation between the central retinal artery collapse pressure measured in relative units and the diastolic brachial blood pressure in the control study group. Correlation coefficient: 0.77; equation of the regression line: diastolic arterial blood pressure $=0.46 \times$ (central retinal artery collapse pressure $)+42.7$; $\mathrm{p}<0.001$.

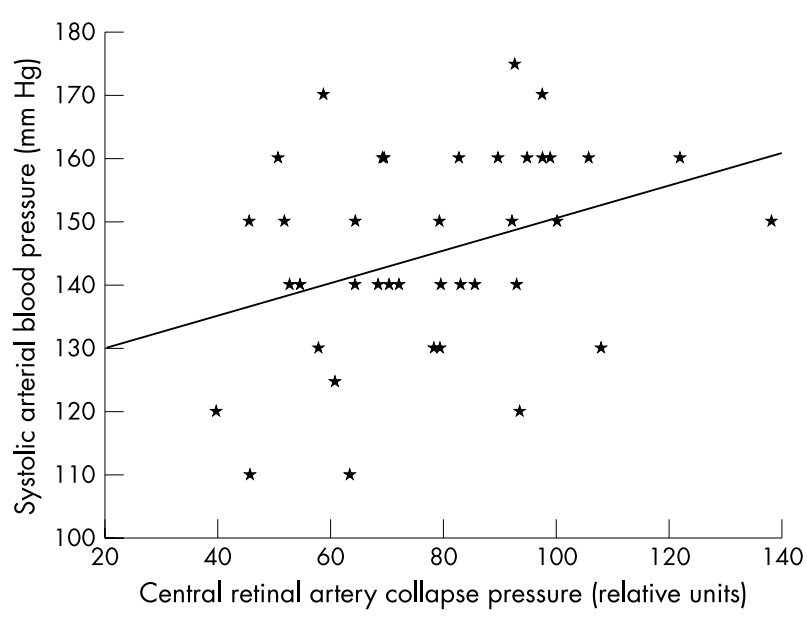

Figure 2 Scattergram showing the correlation between the central retinal artery collapse pressure measured in relative units and the systolic brachial blood pressure in the control study group. Correlation coefficient: 0.35 ; equation of the regression line: systolic arterial blood pressure $=0.26 \times$ (central retinal artery collapse pressure $)+125.1$; $\mathrm{p}=0.029$.

correlated with the diastolic blood pressure (fig 1), the mean arterial blood pressure, and systolic blood pressure (fig 2; table 1). In a primary statistical analysis step, the central retinal artery collapse pressure was statistically independent of age $(p=0.47)$. This result was confirmed in a multiple linear regression analysis $(p=0.18)$ taking into account age and diastolic blood pressure. Additionally, the central retinal artery collapse pressure was statistically independent of gender (table 1).

The correlation coefficients for the relation between central retinal artery collapse pressure and diastolic blood pressure were higher for left eyes (correlation coefficient $r=0.79$; $\mathrm{p}<0.001)$ than for right eyes $(r=0.59 ; \mathrm{p}<0.001)$. The same holds true for the relationship between central retinal artery collapse pressure and systolic blood pressure $(r=0.20$; $\mathrm{p}=0.32$ (not significant) (right eyes) $v r=0.41 ; \mathrm{p}=0.04$ (left eyes)), and for the relationship between central retinal artery collapse pressure and mean arterial blood pressure $(r=0.59 ; \mathrm{p}=0.001$ (right eyes) $v \quad r=0.76 ; \mathrm{p}<0.001 \quad$ (left eyes)).

Collapse pressure of the central retinal vein was not significantly correlated with diastolic or systolic blood pressure, age, and sex (table 1).

If instead of the ophthalmodynamometric measurements obtained in relative units, the sum of ophthalmodynamometric readings (relative units) plus intraocular pressure $(\mathrm{mm} \mathrm{Hg})$ was correlated with arterial blood pressure, the correlation coefficients were generally slightly lower than the coefficients of the correlations between blood pressure measurements and ophthalmodynamometric readings without taking into account IOP (table 1).

In the second study group with ocular or orbital diseases in which the retinal arterial perfusion was not completely reduced, collapse pressure of the central retinal artery was significantly correlated with mean arterial blood pressure (table 1). The correlations between central retinal artery collapse pressure and diastolic blood pressure or systolic blood pressure were marginally significant. The correlation coefficients were generally lower than in the control study group. The central retinal artery collapse pressure was statistically independent of age and sex. Mean central retinal artery collapse pressure was not significantly different from 
Table 1 Correlations between ophthalmodynamometric measurements, blood pressure readings, age, and sex

\begin{tabular}{lrc}
\hline Study group 1 (control) & $\begin{array}{c}\text { Correlation } \\
\text { coefficient }\end{array}$ & p Value \\
\hline Correlation between central retinal & & \\
artery collapse pressure (relative units) with: & & \\
Diastolic arterial BP & 0.77 & $<0.001$ \\
Mean arterial BP & 0.76 & $<0.001$ \\
Systolic arterial BP & 0.35 & 0.029 \\
Age & -0.13 & 0.47 (NS) \\
Sex & & 0.91 (NS) \\
Correlation between central retinal artery & & \\
collapse (relative units) pressure plus & & \\
IOP pressure (mm Hg) with: & 0.71 & $<0.001$ \\
Diastolic arterial BP & 0.67 & $<0.001$ \\
Mean arterial BP & 0.27 & 0.19 (NS) \\
Systolic arterial BP & -0.19 & 0.41 (NS) \\
Age & & 0.31 (NS) \\
Sex & & \\
Correlation between central retinal vein & & \\
collapse pressure (relative units) with: & 0.26 & 0.11 (NS) \\
Diastolic arterial BP & 0.19 & 0.24 (NS) \\
Mean arterial BP & -0.10 & 0.54 (NS) \\
Systolic arterial BP & -0.03 & 0.89 (NS) \\
Age & & 0.42 (NS) \\
Sex & & \\
IOP (mm Hg) with: & 0.18 & 0.39 (NS) \\
Diastolic arterial BP & 0.11 & 0.59 (NS) \\
Mean arterial BP & -0.10 & 0.61 (NS) \\
Systolic arterial BP & -0.19 & 0.41 (NS) \\
Age & & 1.0 (NS) \\
Sex & & \\
\hline Study group 2 (retinal or orbital diseases) & & \\
\hline
\end{tabular}

Study group 2 (retinal or orbital diseases)

Correlation between central retinal artery

collapse pressure (relative units) with:

Diastolic arterial BP 0.28

Mean arterial BP $\quad 0.31$

$\begin{array}{lr}\text { Systolic arterial BP } & 0.23 \\ \text { Age } & -0.04\end{array}$

Sex

Correlation between central retinal artery

collapse pressure (relative units) plus IOP

$(\mathrm{mm} \mathrm{Hg})$ with:

Diastolic arterial BP

Mean arterial $B P$

Systolic arterial BP

Age

0.27

0.15 (NS)

0.43 (NS)

0.46 (NS)

0.06 (NS)

Collapse pressure (relative units) with:

Diastolic arterial BP

0.52 (NS)

Mean arterial BP

Systolic arterial BP

0.11

0.06

0.73 (NS)

0.69 (NS)

0.31 (NS)

0.46 (NS)

$-0.18$

Correlation between central retinal vein

collapse pressure (relative units) plus IOP $(\mathrm{mm} \mathrm{Hg}$ ) with:

Mean arterial BP

Systolic arterial BP

Age

0.01

$-0.03$

0.96 (NS)

0.86 (NS)

0.61 (NS)

0.25 (NS)

0.06 (NS)

Study group 3 (retinal occlusive diseases)

Correlation between central retinal artery

collapse pressure (relative units) with

Diastolic a teria BP

Mean arterial BP

Systolic arterial BP

Age

$-0.24$

Sex

Correlation between central retinal vein

collapse pressure (relative units) with:

Diastolic arterial BP

Mean arterial BP

Systolic arterial BP

Age

Sex
$-0.10$

$-0.17$

$-0.31$

$-0.36$

0.78 (NS)

0.61 (NS)

0.36 (NS)

0.28 (NS)

0.70 (NS)

$-0.17$

0.63 (NS)

0.87 (NS)

$\begin{array}{ll}0.64 & 0.05 \text { (NS) } \\ 0.05 & 0.98 \text { (NS) }\end{array}$

0.10 (NS)
BP, blood pressure; IOP, intraocular pressure; NS, not significant. the mean central retinal artery collapse pressure in the control study group $(74.5$ (SD 19.2) relative units $v 73.2$ (21.0) relative units; $\mathrm{p}=0.78)$.

The central retinal vein collapse pressure was statistically independent of diastolic blood pressure, systolic blood pressure, age, and sex (table 1).

In the third study group, consisting of patients with central retinal artery occlusion, branch retinal artery occlusion, and ischaemic ophthalmopathy, central retinal artery collapse pressure and central retinal vein collapse pressure were not significantly correlated with arterial blood pressure measurements, age, and sex (table 1). The central arterial collapse pressure was significantly lower in this study group than in the control study group (50.5 (SD 31.8) relative units $v 74.5$ (SD 19.2) relative units; $\mathrm{p}=0.008$ ).

\section{DISCUSSION}

The results of the present study suggest that the central retinal artery collapse pressure as determined by the new ophthalmodynamometric technique is highly significantly correlated with the diastolic blood pressure and, to a lesser degree, with the systolic blood pressure (figs 1 and 2). The reason for the discrepancy in the correlation coefficients between the diastolic blood pressure and the systolic blood pressure may be that it was the diastolic value of the central retinal artery collapse pressure which was measured. The correlation between the ophthalmodynamometric measurements and the brachial arterial measurements were best for patients without ocular diseases primarily related to the retina or optic nerve. The correlation was worst or nonexistent for eyes with retinal arterial occlusive diseases such as central retinal artery occlusion, branch retinal artery occlusion, and ischaemic ophthalmopathy due to a stenosis of the carotid artery. The reason is that in eyes with retinal arterial occlusive diseases the retinal arterial collapse pressure is almost zero, destroying any correlation with systemic blood pressure measurements.

Interestingly, the correlation coefficients were higher for left eyes than for right eyes. The reason may be that the ophthalmodynamometric examination changed the arterial blood pressure due to the oculocardiac reflex, so that the arterial blood pressure measured after ophthalmodynamometry was more closely related to ophthalmodynamometric examination of the left eye which was measured last.

There are limitations to the study. The target parameter for ophthalmodynamometry is the IOP when the retinal vessels start pulsating. It is the sum of the IOP before the start of the examination plus the pressure exerted onto the globe by the ophthalmodynamometric contact lens. The IOP change produced by the ophthalmodynamometric contact lens, however, was not measured in absolute measurement units such as mm Hg. It was, therefore, a compromise to calculate a parameter as sum of IOP measurements obtained before the start of the examination, plus the relative measurements values given by the ophthalmodynamometer. Interestingly, the correlation coefficients were slightly lower for the sum of ophthalmodynamometric measurements plus IOP readings than for the ophthalmodynamometric measurements alone (table 1). Future studies are warranted which may evaluate how the relative measurements obtained by the ophthalmodynamometer can be transferred into data of change in IOP produced by the pressure of the ophthalmodynamometer and given in $\mathrm{mm} \mathrm{Hg}$. Another limitation of the study may be that the second study group and third study group were heterogeneous. The control study group, however, was rather homogenous and included patients and subjects with refractive problems or cataract. It may reflect the majority of patients attending the office of an ophthalmologist, whereas patients with endocrine orbitopathy, retinal venous 
stasis syndrome, and retinal arterial occlusive diseases represent a minority of patients seeking the advice of an ophthalmologist. The division of the whole population group into three subgroups enabled us to show that correlations between the ophthalmodynamometric measurements and the blood pressure determinations varied between study groups, with the highest correlation coefficients for the control study group and no correlations at all for the retinal arterial occlusive group. It suggests clinically, that for patients without diseases of the retina or optic nerve, the ophthalmodynamometric technique may provide indication of arterial blood pressure. In patients with retinal or optic nerve diseases, the ophthalmodynamometric measurements are less indicative for arterial blood pressure than they are for patients without retinal or optic nerve diseases. Finally, for patients with retinal arterial occlusive diseases, the ophthalmodynamometric technique is not useful to get any indirect information about the arterial blood pressure.

From a practical point of view, the results of the present study suggest that by simple, minimally invasive ophthalmoscopy using a conventional Goldmann contact lens with a pressure sensor mounted into its holding grip, a rough estimation of the systemic blood pressure may be possible. If during a routine ophthalmoscopic examination of a patient, the central retinal artery collapse pressure is found to be abnormally high, arterial blood pressure should be measured in an attempt not to miss the diagnosis of arterial hypertension. If during a routine ophthalmoscopic examination of a patient, the central retinal artery collapse pressure is measured to be abnormally low, a stenosis of the carotid artery or other cerebrovascular abnormalities may be suspected. It holds true especially if a discrepancy exists between a normal arterial blood pressure and an abnormally low central retinal artery collapse pressure. Additionally, blood pressure values should be taken into account for ophthalmodynamometric measurements. A low ophthalmodynamometric reading of the central retinal artery collapse pressure in a patient without retinal or orbital diseases may not necessarily point towards a stenosis of the carotid artery but may be explainable by a low systemic arterial blood pressure.

In conclusion, ophthalmodynamometric determination of the central retinal artery collapse pressure, performed during a routine Goldmann contact lens ophthalmoscopy, may be useful for an approximate estimation of the systemic blood pressure. The correlation coefficients for the relations between the ophthalmodynamometric measurements and the brachial blood pressure measurements are highest for patients with ocular diseases unrelated to the retina or optic nerve, such as cataract or refractive problems. The correlation coefficients are lower for eyes with diseases affecting the blood vessels of the retina or orbit. The correlations do not exist for eyes with retinal arterial occlusive diseases such as central retinal artery occlusion, branch retinal artery occlusion, and ischaemic ophthalmopathy. Using the new and simple device, ophthalmodynamometry for measurement of the central retinal artery collapse pressure may give some information on the systemic blood pressure as well as on the status of oculoafferent blood vessels.

Proprietary interest: none.

Correspondence to: Dr J Jonas, Universitäts-Augenklinik, TheodorKutzer-Ufer 1-3, 68167 Mannheim, Germany;

Jost.Jonas@augen.ma.uni-heidelberg.de

Accepted for publication 1 September 2003

\section{REFERENCES}

1 Duke-Elder WS. The ocular circulation: its normal pressure relationships and their physiological significance. Br J Ophthalmol 1926;10:513-72.

2 Wunsh SE. Ophthalmodynamometry. N Engl J Med 1969;281:446.

3 Hedges TR, Weinstein JD, Kassell NF, et al. Correlation of ophthalmodynamometry with ophthalmic artery pressure in the rhesus monkey. Am J Ophthalmol 1965;60:1098-101.

4 Galin MA, Baras I, Dodick JM. Semiautomated suction ophthalmodynamometry. Am J Ophthalmol 1969;68:237-40

5 Best $M$, Blumenthal $M$, Futterman $\mathrm{HA}$, et al. Critical closure of intraocular blood vessels. Arch Ophthalmol 1969;82:385-92.

6 Van der Werff TJ. The pressure measured in ophthalmodynamometry. Arch Ophthalmol 1972;87:290-2.

7 Rios-Montenegro EN, Anderson DR, David NJ. Intracranial pressure and ocular hemodynamics. Arch Ophthalmol 1973;89:52-8.

8 Yablonski ME. A new fundus lens ophthalmodynamometer. Am J Ophthalmol 1978;86:644-7.

9 9.Krieglstein GK, da Silva FA. Comparative measurements of the ophthalmic arterial pressure using the Mikuni dynamometer and the Stepanikarteriotonograph. Albrecht Von Graefes Arch Klin Exp Ophthalmol 1979;212:77-91.

10 Lovasik JV, Kothe AC, Kergoat H. Comparison of noninvasive methods to derive the mean central retinal artery pressure in man. Optom Vis $\mathrm{Sci}$ 1993;70:1005-101.

11 Morgan WH, Yu DY, Cooper RL, et al. Retinal artery and vein pressures in the dog and their relationship to aortic, intraocular and cerebrospinal fluid pressures. Microvasc Res 1997;53:211-21.

12 Entenmann B, Robert YC, Pirani P, et al. Contact lens tonometry-application in humans. Invest Ophthalmol Vis Sci 1997;38:2447-51.

13 Morgan WH, Yu DY, Alder VA, et al. Relation between pressure determined by ophthalmodynamometry and aortic pressure in the dog. BrJ Ophthalmol 1998;82:821-5.

14 Firsching R, Schutze M, Motschmann M, et al. Venous opthalmodynamometry: a noninvasive method for assessment of intracranial pressure. J Neurosurg 2000;93:33-6.

15 Motschmann M, Muller C, Kuchenbecker J, et al. Ophthalmodynamometry: a reliable method for measuring intracranial pressure. J Neurosurg 2000;93:33-6.

16 Hartmann K, Meyer-Schwickerath R. Measurement of venous outflow pressure in the central retinal vein to evaluate intraorbital pressure in Graves' ophthalmopathy: a preliminary report. Strabismus 2000;8:187-93.

17 Jonas JB, Niessen A. Ophthalmodynamometric diagnosis of unilateral ischemic ophthalmopathy. Am J Ophthalmol 2002;134:91 1-12.

18 Jonas JB, Harder B. Ophthalmodynamometric estimation of cerebrospinal fluid pressure in pseudotumor cerebri. Br J Ophthalmol 2003;87:361-2.

19 Jonas JB. Ophthalmodynamometric assessment of the central retinal vein collapse pressure in eyes with retinal vein stasis or occlusion. Graef Arch Clin Exp Ophthalmol 2003;241:367-70.

20 Jonas JB. Reproducibility of ophthalmodynamometric measurements of the central retinal artery and vein collapse pressure. $\mathrm{Br} J$ Ophthalmol 2003;87:577-9.

21 Jonas JB, Groden C. Spontaneous carotid-cavernous sinus fistula diagnosed by ophthalmodynamometry. Acta Ophthalmol 2003;81:419-20.

22 Jonas JB. Ophthalmodynamometry in eyes with dilated episcleral veins. J Glaucoma 2003;12:285-7.

23 Jonas JB. Central retinal artery and vein pressure in patients with chronic open angle glaucoma. Br J Ophthalmol 2003;87:949-51.

24 Jonas JB. Ophthalmodynamometric measurement of orbital tissue pressure in thyroid-associated orbitopathy. Acta Ophthalmol 2004 (in press).

25 Jonas JB. Retinal arterial collapse pressure in eyes with retinal arteria occlusive diseases. Br J Ophthalmol 2004 (in press). 